\title{
SUMMATION FORMULAS DERIVED FROM THE SRIVASTAVA'S TRIPLE HYPERGEOMETRIC SERIES $H_{C}$
}

\author{
Yong Sup Kim, Arjun kumar Rathie, And Junesang Choi
}

\begin{abstract}
Srivastava noticed the existence of three additional complete triple hypergeometric functions $H_{A}, H_{B}$ and $H_{C}$ of the second order in the course of an extensive investigation of Lauricella's fourteen hypergeometric functions of three variables. In 2004, Rathie and Kim obtained four summation formulas containing a large number of very interesting reducible cases of Srivastava's triple hypergeometric series $H_{A}$ and $H_{C}$. Here we are also aiming at presenting two unified summation formulas (actually, including 62 ones) for some reducible cases of Srivastava's $H_{C}$ with the help of generalized Dixon's theorem and generalized Whipple's theorem on the sum of a ${ }_{3} F_{2}$ obtained earlier by Lavoie et al.. Some special cases of our results are also considered.
\end{abstract}

\section{Introduction}

The triple hypergeometric series $H_{A}$ and $H_{C}$ introduced by Srivastava (see [9, pp. 68-69, Eqs. (36) and (38)]) are defined as follows:

$$
\begin{aligned}
& H_{A}\left(\alpha, \beta, \beta^{\prime} ; \gamma, \gamma^{\prime} ; x, y, z\right)=\sum_{m, n, p=0}^{\infty} \frac{(\alpha)_{m+p}(\beta)_{m+n}\left(\beta^{\prime}\right)_{n+p}}{(\gamma)_{m}\left(\gamma^{\prime}\right)_{n+p} m ! n ! p !} x^{m} y^{n} z^{p} \\
& (|x|<r,|y|<s,|z|<t, \text { and } r+s+t=1+s t) ; \\
& H_{C}\left(\alpha, \beta, \beta^{\prime} ; \gamma ; x, y, z\right)=\sum_{m, n, p=0}^{\infty} \frac{(\alpha)_{m+p}(\beta)_{m+n}\left(\beta^{\prime}\right)_{n+p}}{(\gamma)_{m+n+p} m ! n ! p !} x^{m} y^{n} z^{p} \\
& (|x|<1,|y|<1, \text { and }|z|<1),
\end{aligned}
$$

where $(\alpha)_{n}$ denotes the Pochhammer symbol defined, in terms of the wellknown Gamma function $\Gamma(z)$, by

$$
(\alpha)_{n}=\frac{\Gamma(\alpha+n)}{\Gamma(\alpha)} \quad(n=0,1,2, \ldots) .
$$

Received September 21, 2007.

2000 Mathematics Subject Classification. Primary 33C20, 33C60, 39A10; Secondary 33C70, 33C65.

Key words and phrases. triple hypergeometric series $H_{A}$ and $H_{C}$, Appell's function, generalized Dixon's theorem, generalized Whipple's theorem. 
We recall only two identities for the Pochhammer symbol (see [8, pp. 6-8]):

$$
(\alpha)_{m}(\alpha+m)_{n}=(\alpha)_{m+n}
$$

and

$$
\frac{\Gamma(\alpha-n)}{\Gamma(\alpha)}=\frac{(-1)^{n}}{(1-\alpha)_{n}}
$$

In [7], Rathie and Kim obtained a large number of summation formulas for the series $H_{A}$ and $H_{C}$. In fact they have presented explicit Gamma functionexpressions for the following cases:

(i)

$$
\begin{gathered}
H_{A}\left(\alpha, \beta, \beta^{\prime} ; 1+\beta-\alpha+\frac{1}{2} \beta^{\prime}+i, 1+\beta+\beta^{\prime}-\alpha ;-1,1,-1\right) \\
(i=0, \pm 1, \pm 2, \pm 3, \pm 4, \pm 5)
\end{gathered}
$$

(ii)

$$
\begin{gathered}
H_{A}\left(\alpha, \beta, \beta^{\prime} ; \frac{1}{2}\left(1+\alpha+\beta-\frac{1}{2} \beta^{\prime}+i\right), 1+\beta+\beta^{\prime}-\alpha ; \frac{1}{2}, 1,-1\right) \\
(i=0, \pm 1, \pm 2, \pm 3, \pm 4, \pm 5) ;
\end{gathered}
$$

(iii)

$$
\begin{gathered}
H_{A}\left(\alpha, \beta, 2 \alpha+2 \beta-2-2 i ; \gamma, 3 \beta+\alpha-2 i-1 ; \frac{1}{2}, 1,-1\right) \\
(i=0, \pm 1, \pm 2, \pm 3, \pm 4, \pm 5)
\end{gathered}
$$

(iv)

$$
\begin{gathered}
H_{C}(\alpha, \beta, \beta+i+j ; 3 \beta+2 j+i ; 1,1,1) \\
(i=0, \pm 1, \pm 2) .
\end{gathered}
$$

These results are derived with the help of generalized Kummer's theorem, generalized Gauss' second theorem, generalized Bailey's theorem and generalized Watson's theorem obtained earlier by Lavoie et al. [4, 5].

Here we are aiming at providing 62 further new reducible cases in the form of two unified formulas for the triple hypergeometric series $H_{C}$ by using, mainly, generalized Dixon's theorem and generalized Whipple's theorem obtained earlier by Lavoie et al. [5, 6]. Those easily established results in this paper may be interesting and useful.

\section{Results required}

In this section we just recall some known results which will be used for our present investigation. Appell and Kampé de Fériet [9, p. 55, Eq. (15)]:

$$
\begin{gathered}
F_{1}\left(a, b, b^{\prime} ; c ; 1,1\right)=\frac{\Gamma(c) \Gamma\left(c-a-b-b^{\prime}\right)}{\Gamma(c-a) \Gamma\left(c-b-b^{\prime}\right)} \\
\left(\Re\left(c-a-b-b^{\prime}\right)>0 ; c \neq 0,-1,-2, \ldots\right) .
\end{gathered}
$$


Generalized Dixson's theorem [6]:

$(2.2)$

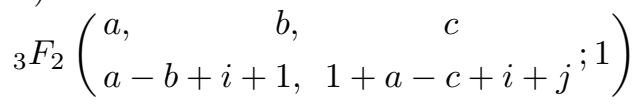

$$
\begin{aligned}
& =\frac{2^{-2 c+i+j} \Gamma(a-b+i+1) \Gamma(1+a-c+i+j) \Gamma\left(b-\frac{1}{2}|i|-\frac{1}{2} i\right) \Gamma\left(c-\frac{1}{2}(i+j+|i+j|)\right)}{\Gamma(b) \Gamma(c) \Gamma(a-2 c+i+j+1) \Gamma(a-b-c+i+j+1)} \\
& \times\left\{A_{i, j} \frac{\Gamma\left(\frac{1}{2} a-c+\frac{1}{2}+\left[\frac{i+j+1}{2}\right]\right) \Gamma\left(\frac{1}{2} a-b-c+1+i+\left[\frac{j+1}{2}\right]\right)}{\Gamma\left(\frac{1}{2} a+\frac{1}{2}\right) \Gamma\left(\frac{1}{2} a-b+1+\left[\frac{i}{2}\right]\right)}\right. \\
& \left.+B_{i, j} \frac{\Gamma\left(\frac{1}{2} a-c+1+\left[\frac{i+j}{2}\right]\right) \Gamma\left(\frac{1}{2} a-b-c+\frac{3}{2}+i+\left[\frac{j}{2}\right]\right)}{\Gamma\left(\frac{1}{2} a\right) \Gamma\left(\frac{1}{2} a-b+\frac{1}{2}+\left[\frac{i+1}{2}\right]\right)}\right\} \\
& (\Re(a-2 b-2 c)>-2-2 i-j ; i=-3,-2,-1,0,1,2 ; j=0,1,2,3) .
\end{aligned}
$$

Here and in what follows, $[x]$ is the greatest integer less than or equal to $x$ and $|x|$ denotes the usual absolute value of $x$. The coefficients $A_{i, j}$ and $B_{i, j}$ are given in the tables at the end of the paper [6].

It is interesting to observe here that if $f_{i, j}$ is the left-hand side of $(2.2)$, the natural symmetry

$$
f_{i, j}(a, b, c)=f_{i+j,-j}(a, c, b)
$$

makes it possible to extend the result to $j=-1,-2,-3$.

The special case of (2.2) when $i=j=0$ reduces, immediately, to the following classical Dixon's theorem [1]:

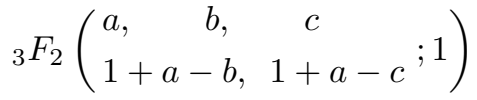

$$
\begin{aligned}
& =\frac{\Gamma\left(1+\frac{1}{2} a\right) \Gamma(1+a-b) \Gamma(1+a-c) \Gamma\left(1+\frac{1}{2} a-b-c\right)}{\Gamma(1+a) \Gamma\left(1+\frac{1}{2} a-b\right) \Gamma\left(1+\frac{1}{2} a-c\right) \Gamma(1+a-b-c)}
\end{aligned}
$$

provided $\Re(a-2 b-2 c)>-2$.

Generalized Whipple's theorem [5]:

$$
\begin{aligned}
& { }_{3} F_{2}\left(\begin{array}{c}
a, b, c \\
e, f
\end{array} ;\right) \\
= & \frac{2^{-2 a+i+j} \Gamma(e) \Gamma(f) \Gamma\left(c-\frac{1}{2}(j+|j|)\right) \Gamma\left(e-c-\frac{1}{2}(i+|i|)\right) \Gamma\left(a-\frac{1}{2}(i+j+|i+j|)\right)}{\Gamma(a) \Gamma(c) \Gamma(e-a) \Gamma(f-a) \Gamma(e-c)} \\
& \times\left\{C_{i, j} \frac{\Gamma\left(\frac{1}{2} e-\frac{1}{2} a+\frac{1}{4}\left(1-(-1)^{i}\right)\right) \Gamma\left(\frac{1}{2} f-\frac{1}{2} a\right)}{\Gamma\left(\frac{1}{2} e+\frac{1}{2} a-\frac{1}{2} i+\left[\frac{-j}{2}\right]\right) \Gamma\left(\frac{1}{2} f+\frac{1}{2} a-\frac{1}{2} i+\frac{(-1)^{j}}{4}\left((-1)^{i}-1\right)+\left[-\frac{j}{2}\right]\right)}\right. \\
& \left.+D_{i, j} \frac{\Gamma\left(\frac{1}{2} e-\frac{1}{2} a+\frac{1}{4}\left(1+(-1)^{i}\right)\right) \Gamma\left(\frac{1}{2} f-\frac{1}{2} a+\frac{1}{2}\right)}{\Gamma\left(\frac{1}{2} e+\frac{1}{2} a-\frac{1}{2}-\frac{1}{2} i+\left[-\frac{j}{2}+\frac{1}{2}\right]\right) \Gamma\left(\frac{1}{2} f+\frac{1}{2} a-\frac{1}{2}-\frac{1}{2} i+\frac{(-1)^{j}}{4}\left(1-(-1)^{i}\right)+\left[-\frac{j}{2}+\frac{1}{2}\right]\right)}\right\},
\end{aligned}
$$


where $a+b=i+j+1$ and $e+f=2 c+i+1, i, j$ takes values in a subset of $i, j=0, \pm 1, \pm 2, \pm 3$, and the coefficients $C_{i, j}$ and $D_{i, j}$ are given in the tables in $[5]$.

The special case $i=j=0$ of (2.5) yields the following classical Whipple's theorem [1]:

$$
{ }_{3} F_{2}\left(\begin{array}{lll}
a, & b, & c \\
e, & f
\end{array}\right)=\frac{2^{-2 c+1} \pi \Gamma(e) \Gamma(f)}{\Gamma\left(\frac{1}{2} e+\frac{1}{2} a\right) \Gamma\left(\frac{1}{2} f+\frac{1}{2} a\right) \Gamma\left(\frac{1}{2} e+\frac{1}{2} b\right) \Gamma\left(\frac{1}{2} f+\frac{1}{2} b\right)},
$$

where $a+b=1$ and $e+f=2 c+1$.

\section{Main summation formulas}

The 62 summation formulas established in this paper can be presented in the following two unified forms. If $\Re(2 \beta-\alpha)>2 i+j$ for convergence, we have

$$
\begin{aligned}
& H_{C}(\alpha, \beta, 4 \beta-\alpha-1-3 i-j ; 3 \beta-2 i-j ; 1,1,1) \\
= & \frac{2^{4 \beta-2 \alpha-3 i-j-2} \Gamma(2 \beta-i) \Gamma\left(\beta-\frac{i}{2}-\frac{|i|}{2}\right) \Gamma(1+i-2 \beta)}{\Gamma(\beta) \Gamma(\beta-i) \Gamma(1+\alpha-2 \beta+2 i+j)} \\
& \times \frac{\Gamma(3 \beta-2 i-j) \Gamma\left(1+\alpha-2 \beta+\frac{3}{2} i+\frac{1}{2} j-\frac{1}{2}|i+j|\right)}{\Gamma(2 \beta-\alpha-2 i-j) \Gamma(4 \beta-\alpha-1-3 i-j)} \\
& \times\left\{\bar{A}_{i, j} \frac{\Gamma\left(\beta-\frac{1}{2} \alpha-i-j+\left[\frac{j+1}{2}\right]\right) \Gamma\left(2 \beta-\frac{1}{2} \alpha-\frac{1}{2}-2 i-j+\left[\frac{i+j+1}{2}\right]\right)}{\Gamma\left(\frac{1}{2} \alpha+\frac{1}{2}\right) \Gamma\left(\frac{1}{2} \alpha-\beta+1+\left[\frac{i}{2}\right]\right)}\right. \\
& \left.\quad+\bar{B}_{i, j} \frac{\Gamma\left(\beta-\frac{1}{2} \alpha+\frac{1}{2}-i-j+\left[\frac{j}{2}\right]\right) \Gamma\left(2 \beta-\frac{1}{2} \alpha-2 i-j+\left[\frac{i+j}{2}\right]\right)}{\Gamma\left(\frac{1}{2} \alpha\right) \Gamma\left(\frac{1}{2} \alpha-\beta+\frac{1}{2}+\left[\frac{i+1}{2}\right]\right)}\right\},
\end{aligned}
$$

where $i=0, \pm 1, \pm 2, \pm 3, j=0,1,2,3$, and the coefficients $\bar{A}_{i, j}$ and $\bar{B}_{i, j}$ are obtained from the tables of $A_{i j}$ and $B_{i j}$ in (2.2) by replacing $a, b$, and $c$ by $\alpha$, $\beta$, and $1+\alpha-2 \beta+2 i+j$, respectively. Moreover, in view of the observation (2.3), the results in $(3.1)$ can be extended to $j=-1,-2,-3$.

$$
\begin{aligned}
& H_{C}\left(\alpha, 1-\alpha+i+j, \beta^{\prime} ; \frac{3}{2}+i+\frac{1}{2} j ; 1,1,1\right) \\
= & \frac{2^{-2 \alpha+i+j} \Gamma\left(\frac{3}{2}+i+\frac{1}{2} j\right) \Gamma\left(\frac{1}{2}-\frac{1}{2} j-\beta^{\prime}\right) \Gamma\left(\frac{1}{2}+\frac{1}{2} j+\beta^{\prime}\right)}{\Gamma\left(\frac{1}{2}-\frac{1}{2} j\right) \Gamma\left(\frac{1}{2}+\frac{1}{2} j\right) \Gamma(\alpha)} \\
& \times \frac{\Gamma\left(\frac{1}{2}-\frac{1}{2}|j|\right) \Gamma\left(1+\frac{1}{2} i-\beta^{\prime}-\frac{1}{2}|i|\right) \Gamma\left(\alpha-\frac{1}{2}(i+j+|i+j|)\right)}{\Gamma\left(\frac{3}{2}+i+\frac{1}{2} j-\beta^{\prime}-\alpha\right) \Gamma\left(1-\beta^{\prime}+i\right) \Gamma\left(\frac{1}{2}+\frac{1}{2} j+\beta^{\prime}-\alpha\right)} \\
& \times\left\{\bar{C}_{i, j} \frac{\Gamma\left(\frac{1}{4}+\frac{1}{4} j+\frac{1}{2} \beta^{\prime}-\frac{1}{2} \alpha\right) \Gamma\left(\frac{3}{4}+\frac{1}{2} i+\frac{1}{4} j-\frac{1}{2} \beta^{\prime}-\frac{1}{2} \alpha+\frac{1}{4}\left(1-(-1)^{i}\right)\right)}{\Gamma\left(\frac{3}{4}+\frac{1}{4} j-\frac{1}{2} \beta^{\prime}+\frac{1}{2} \alpha+\left[-\frac{j}{2}\right]\right) \Gamma\left(\frac{1}{2} \alpha+\frac{1}{4}+\frac{1}{4} j-\frac{1}{2} i+\frac{1}{2} \beta^{\prime}+\frac{(-1)^{j}}{4}\left((-1)^{i}-1\right)+\left[-\frac{j}{2}\right]\right)}\right. \\
& \left.+\bar{D}_{i, j} \frac{\Gamma\left(\frac{3}{4}+\frac{1}{4} j+\frac{1}{2} \beta^{\prime}-\frac{1}{2} \alpha\right) \Gamma\left(\frac{3}{4}+\frac{1}{2} i+\frac{1}{4} j-\frac{1}{2} \beta^{\prime}-\frac{1}{2} \alpha+\frac{1}{4}\left(1+(-1)^{i}\right)\right)}{\Gamma\left(\frac{1}{4}+\frac{1}{4} j-\frac{1}{2} \beta^{\prime}+\frac{1}{2} \alpha+\left[-\frac{j}{2}+\frac{1}{2}\right]\right) \Gamma\left(\frac{1}{2} \alpha+\frac{1}{2} \beta^{\prime}+\frac{1}{4} j-\frac{1}{2} i-\frac{1}{4}+\frac{(-1)^{j}}{4}\left(1-(-1)^{i}\right)+\left[-\frac{j}{2}+\frac{1}{2}\right]\right)}\right\},
\end{aligned}
$$


where $i, j=0, \pm 1, \pm 2, \pm 3$, and the coefficients $\bar{C}_{i, j}$ and $\bar{D}_{i, j}$ are obtained from the tables of $C_{i j}$ and $D_{i j}$ in (2.5) by replacing $a, b, c, e$, and $f$ by $\alpha, 1-\alpha+i+j$, $\frac{1}{2}+\frac{1}{2} j, \frac{3}{2}+i+\frac{1}{2} j-\beta^{\prime}$, and $\frac{1}{2}+\frac{1}{2} j+\beta^{\prime}$, respectively.

Now let us start with the proof of (3.1). By using the definition of $H_{C}$ in (1.2), we have

$$
\begin{aligned}
H_{C}: & =H_{C}(\alpha, \beta, 4 \beta-\alpha-1-3 i-j ; 3 \beta-2 i-j ; 1,1,1) \\
& =\sum_{m, n, p=0}^{\infty} \frac{(\alpha)_{m+n}(\beta)_{m+p}(4 \beta-\alpha-1-3 i-j)_{n+p}}{(3 \beta-2 i-j)_{m+n+p} m ! n ! p !},
\end{aligned}
$$

which, upon using (1.3), becomes

$$
\begin{aligned}
H_{C} & =\sum_{m, n, p=0}^{\infty} \frac{(\alpha)_{m}(\alpha+m)_{n}(\beta)_{m}(\beta+m)_{p}(4 \beta-\alpha-1-3 i-j)_{n+p}}{(3 \beta-2 i-j)_{m}(3 \beta-2 i-j+m)_{n+p} m ! n ! p !} \\
& =\sum_{m=0}^{\infty} \frac{(\alpha)_{m}(\beta)_{m}}{(3 \beta-2 i-j)_{m} m !} \sum_{n, p=0}^{\infty} \frac{(\alpha+m)_{n}(\beta+m)_{p}(4 \beta-\alpha-1-3 i-j)_{n+p}}{(3 \beta-2 i-j+m)_{n+p} n ! p !} .
\end{aligned}
$$

By making use of the Apell function of two variables (see [9, p. 53, Eq. (4)] defined by:

$$
F_{1}\left[a, b, b^{\prime} ; c ; x, y\right]=\sum_{m, n=0}^{\infty} \frac{(a)_{m+n}(b)_{m}\left(b^{\prime}\right)_{n}}{(c)_{m+n} m ! n !} x^{m} y^{n}
$$

in (3.4), we obtain

$$
\begin{aligned}
& H_{C} \\
= & \sum_{m=0}^{\infty} \frac{(\alpha)_{m}(\beta)_{m}}{(3 \beta-2 i-j)_{m} m !} F_{1}(4 \beta-\alpha-1-3 i-j, \alpha+m, \beta+m ; 3 \beta-2 i-j+m ; 1,1) .
\end{aligned}
$$

If we use the result (2.1), we have

$$
H_{C}=\sum_{m=0}^{\infty} \frac{(\alpha)_{m}(\beta)_{m} \Gamma(3 \beta-2 i-j) \Gamma(1+i-2 \beta-m)}{\Gamma(1+\alpha-\beta+i+m) \Gamma(2 \beta-\alpha-2 i-j-m) m !} .
$$

By employing (1.4) in (3.6), we get

$$
H_{C}=\frac{\Gamma(3 \beta-2 i-j) \Gamma(1+i-2 \beta)}{\Gamma(1+\alpha-\beta+i) \Gamma(2 \beta-\alpha-2 i-j)} \sum_{m=0}^{\infty} \frac{(\alpha)_{m}(\beta)_{m}(1+\alpha-2 \beta+2 i+j)_{m}}{(1+\alpha-\beta+i)_{m}(2 \beta-i)_{m} m !},
$$

which is rewritten as follows:

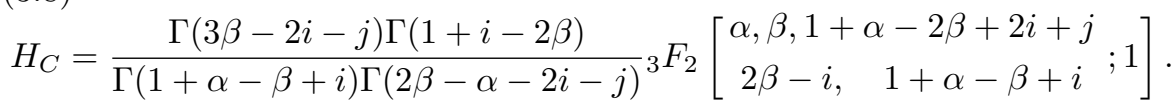


Now if we use the generalized Dixon's theorem (2.2) with $a=\alpha, b=\beta$, $c=1+\alpha-2 \beta+2 i+j(i=-3,-2,-1,0,1,2$, and $j=0,1,2)$, and after a little simplification, we arrive at the right-hand side of (3.1).

For the proof of the result (3.2), proceeding in the same manner as in the proof of (3.1), we get

$$
\begin{aligned}
& H_{C}\left(\alpha, 1-\alpha+i+j, \beta^{\prime} ; \frac{3}{2}+i+\frac{1}{2} j ; 1,1,1\right)
\end{aligned}
$$

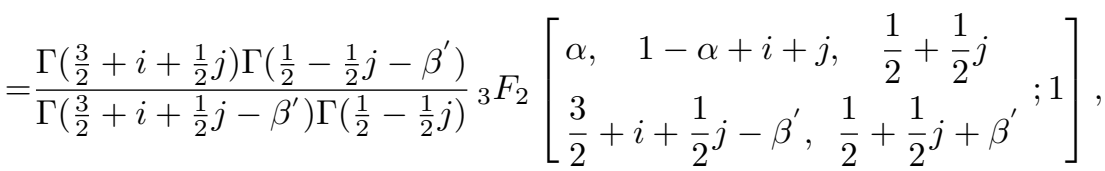

and if we use the generalized Whipple's theorem (2.5) with $a=\alpha, b=1-\alpha+$ $i+j, c=\frac{1}{2}+\frac{1}{2} j, e=\frac{3}{2}+i+\frac{1}{2} j-\beta^{\prime}$, and $f=\frac{1}{2}+\frac{1}{2} j+\beta^{\prime}(i, j=0, \pm 1, \pm 2, \pm 3)$, and after a little simplification, we arrive at the right-hand side of (3.2).

Finally, we present simple special cases of (3.1) and (3.2) by taking $i=j=0$ there:

$$
\begin{aligned}
& H_{C}(\alpha, \beta, 4 \beta-\alpha-1 ; 3 \beta ; 1,1,1) \\
= & \frac{\Gamma\left(\frac{1}{2}\right) \Gamma\left(\frac{1}{2}+\beta\right) \Gamma(3 \beta) \Gamma(1-2 \beta)}{\Gamma\left(\frac{1}{2}+\frac{1}{2} \alpha\right) \Gamma\left(1+\frac{1}{2} \alpha-\beta\right) \Gamma\left(2 \beta-\frac{1}{2} \alpha\right) \Gamma\left(\frac{1}{2}-\frac{1}{2} \alpha+\beta\right)},
\end{aligned}
$$

which is recorded in [2].

$$
\begin{aligned}
& H_{C}\left(\alpha, 1-\alpha, \beta^{\prime} ; \frac{3}{2} ; 1,1,1\right) \\
= & \frac{2^{-2 \alpha-1} \Gamma\left(\frac{1}{2}-\beta^{\prime}\right) \Gamma\left(\frac{1}{2}+\beta^{\prime}\right) \Gamma\left(\frac{1}{4}+\frac{1}{2} \beta^{\prime}-\frac{1}{2} \alpha\right) \Gamma\left(\frac{3}{4}-\frac{1}{2} \beta^{\prime}-\frac{1}{2} \alpha\right)}{\Gamma\left(\frac{3}{2}-\beta^{\prime}-\alpha\right) \Gamma\left(\frac{1}{2}+\beta^{\prime}-\alpha\right) \Gamma\left(\frac{3}{4}-\frac{1}{2} \beta^{\prime}+\frac{1}{2} \alpha\right) \Gamma\left(\frac{1}{2} \alpha+\frac{1}{2} \beta^{\prime}+\frac{1}{4}\right)},
\end{aligned}
$$

which is (presumably) new.

\section{References}

[1] W. N. Bailey, Generalized Hypergeometric Series, Cambridge University Press, Cambridge, 1935

[2] Y. S. Kim, A. K. Rathie, and J. Choi, Note on Srivastava's triple hypergeometric series $H_{A}$ and $H_{C}$, Commun. Korean Math. Soc. 18 (2003), no. 3, 581-586.

[3] G. Lauricella, Sulle funzioni ipergeometriche a piu variabli, Rend. Circ. Mat. Palermo 7 (1893), 111-158.

[4] J. L. Lavoie, F. Grondin, and A. K. Rathie, Generalizations of Watson's theorem on the sum of $a_{3} F_{2}$,Indian J. Math. 34 (1992), no. 1, 23-32.

[5] , Generalizations of Whipple's theorem on the sum of $a_{3} F_{2}$, J. Comput. Appl. Math. 72 (1996), no. 2, 293-300.

[6] J. L. Lavoie, F. Grondin, A. K. Rathie, and K. Arora, Generalizations of Dixon's theorem on the sum of a ${ }_{3} F_{2}$, Math. Comp. 62 (1994), no. 205, 267-276.

[7] A. K. Rathie and Y. S. Kim, Further results on Srivastava's triple hypergeometric series $H_{A}$ and $H_{C}$, Indian J. Pure Appl. Math. 35 (2004), no. 8, 991-1002.

[8] H. M. Srivastava and J. Choi, Series Associated with the Zeta and Related Functions, Kluwer Academic Publishers, Dordrecht, 2001. 
[9] H. M. Srivastava and H. L. Manocha, A Treatise on Generating Functions, Ellis Horwood Ltd., Chichester, New York, 1984.

Yong SuP KIM

Department of Mathematics Education

WONKWANG UNIVERSITY

IKSAN 570-749, KOREA

E-mail address: yspkim@wonkwang.ac.kr

ArJun kumar Rathie

Department of Mathematics Education

Vedant College of Engineering and Technology

TULSI-323021

Dist. Bundi Rajasthan State, India

E-mail address: akrathie@rediffmail.com

JunESANG ChOI

Department of Mathematics Education

DongGuK UNIVERSITY

KYONGJu 780-714, KoreA

E-mail address: junesang@dongguk.ac.kr 